\author{
S. Saghali ${ }^{1}$, F.D. Saei ${ }^{1, *}$, M. Javidi ${ }^{2}$, M.J. $\operatorname{Rad}^{1}$ \\ ${ }^{1}$ Islamic Azad University Tabriz Branch, Tabriz, Iran; \\ ${ }^{2}$ University of Tabriz, Tabriz, Iran \\ (E-mail: stu.s.saghali@iaut.ac.ir,dastmalchi@iaut.ac.ir,mo-javidi@tabriz.ac.ir,Jahangiri@iaut.ac.ir)
}

\title{
Analytical solution of a fractional differential equation in the theory of viscoelastic fluids
}

\begin{abstract}
The aim of this paper is to present analytical solutions of fractional delay differential equations (FDDEs) of an incompressible generalized Oldroyd-B fluid with fractional derivatives of Caputo type. Using a modification of the method of separation of variables the main equation with non-homogeneous boundary conditions is transformed into an equation with homogeneous boundary conditions, and the resulting solutions are then expressed in terms of Green functions via Laplace transforms. This results presented in two condition, in first step when $0 \leq \alpha, \beta \leq \frac{1}{2}$ and in the second step we considered $\frac{1}{2} \leq \alpha, \beta \leq 1$, for each step 1,2 for the unsteady flows of a generalized Oldroyd-B fluid, including a flow with a moving plate, are considered via examples.
\end{abstract}

Keywords: Oldroyd-B fluid, fractional-order partial differential equations, analytical solutions, Delay differential equation, modified separation of variables method, Caputo fractional derivatives.

\section{Introduction}

Many real-world processes can be cast generally in the form of fractional differential systems with integer order (i.e., ordinary differential equations and systems) but there is a growing number of researchers that believe that fractional-differential equations can describe and model and complex physical processes more accurately than the corresponding ordinary differential equations. So, in recent decades the search for analytical and numerical solutions to fractional differential equations has been of considerable interest [1-4]. Fractional differential equations can be applied to the dynamic modeling of non-Newtonian fluids: for example, in the modeling of melting plastics and in the study of emulsion plastics or soft tissue. Practically speaking, there are few Newtonian fluids in reality, so most fluids are of the non-Newtonian type, which means there is no linear relationship between the stress tensor and the deformation tensor [5].

Viscoelastic fluids form an important class of non-Newtonian fluids, which exhibit both elastic and viscous properties. Among them the so-called Oldroyd-B fluid can be used to describe the response of fluids that have a small memory. This means that whenever they flow, these fluids will spend less time to find the first state and stability [6-7]. Due to the wide range of applications of these fluids, considerable attention has been paid to the prediction of the behavior of non-Newtonian fluids. Structural equations that are presented in a constitutive rheological fashion have a fractional calculation, so they are very effective for working with viscoelastic properties [8-9]. The viscoelastic fluid equations in fractional models are obtained by replacing ordinary derivatives with one of many possible definitions of fractional derivatives in the defining equations. In the study of fluids we deal with a phenomenon called delay, which is due to the distance between the sensor and the source of changes arising from e.g., plumbing, measurement slowness, or complex dynamics. Different methods for finding analytical solutions of these type of equations are proposed: an analytical solution for unsteady helical flows is presented by Tong et al in [10]. In Haitao and Mingyu [11] there is a discussion of an Oldroyd-B fluid between two parallel plates. In addition, Fetecau [12-13] developed a generalization of the flow of viscoelastic fluids between two-sided walls. Then Shah [14], Qi [15], Zheng et al [16] and Hayat [17] discussed the generalized flow of an Oldroyd-B fluid under varying conditions. In closing this brief review we mention that Javidi and Heris [18] gave analytical solutions of various forms of such delay equations.

Many events in the natural world can be modeled to form of fractional delay differential equations (FDDEs). FDDEs have important applications in many fields for example technology, economics, biology, medical science,

\footnotetext{
${ }^{*}$ Corresponding author.

E-mail:dastmalchi@iaut.ac.ir
} 
physics and finance [19]. Some numerical methods for FDDEs are introduced in [20-23] and etc. Heris and Javidi [24] proposed a numerical method based on fractional backward differential formulas (FBDF) for solving fractional delay differential equations. Also they found the Green's functions for this equation corresponding to periodic/anti-periodic conditions in terms of the functions of Mittag Leffler type.

In this paper we present analytical solutions for unsteady flows of a generalized Oldroyd-B fluid with constant delay time using Riemann-Liouville fractional derivatives as the defining derivatives. A new separation of variables method [25] and use of Laplace transforms for the Riemann-Liouville fractional derivative are adapted to solve the new governing equation for fractional differential equations with constant delay when applied to viscoelastic fluids.

The paper is structured as follows: in section 2 we recall some basic definitions of fractional calculus; in section 3 we give the derivation of the governing equation; section 4 deals with the method of separation of variables, the Laplace transformation applied to fractional derivatives in two steps $0 \leq \alpha, \beta \leq \frac{1}{2}$ and $\frac{1}{2} \leq \alpha$, $\beta \leq 1$, and the method of solution for each two steps separatively. Finally, in section 5 we give the examples dealing with varying initial conditions by considering two condition for $\alpha$ and $\beta$.

\section{Preliminaries}

In this section we will introduce some of the fundamental definitions.

Defenition 1.1 ([1]). Euler's gamma function is defined by the integral

$$
\Gamma(z)=\int_{0}^{\infty} e^{-t} t^{z-1} d t, \operatorname{Re}(z)>0 .
$$

$C(J, R)$ denotes the Banach space of all continuous functions from $J=[0, T]$ into $R$ with the norm

$$
\|u\|_{\infty}=\sup \{|u(t)|: t \in J\}, \quad T>0 .
$$

$C^{n}(J, R)$ denotes the class of all real valued functions defined on $J=[0, T], T>0$ which have continuous $n$-th order derivatives.

Defenition 1.2 [4]. The fractional integral of order $\alpha>0$ of the function $f \in C(J, R)$ is defined as

$$
I^{\alpha} f(t)=\frac{1}{\Gamma(\alpha)} \int_{0}^{t} \frac{f(s)}{(t-s)^{1-\alpha}} d s, \quad 0<t<T .
$$

Defenition 1.3 [4]. The Riemann-Liouville fractional derivative of order $\alpha>0$ of the function $f \in C(J, R)$ is defined as

$$
{ }^{R L} D^{\alpha} f(t)=\left\{\begin{array}{l}
D^{n} I^{n-\alpha} f(t)=\frac{1}{\Gamma(n-\alpha)}\left(\frac{d^{n}}{d t^{n}}\right) \int_{0}^{t} \frac{f(s)}{(t-s)^{\alpha-n+1}} d s, \\
n-1<\alpha<n, n \in N, \\
f^{(n)}(t), \quad \alpha=n .
\end{array}\right.
$$

Defenition 1.4 [4]. The Caputo fractional derivative of order $\alpha>0$ of the function $f \in C^{n}(J, R)$ is defined as

$$
{ }^{C} D^{\alpha} f(t)=\left\{\begin{array}{rr}
I^{n-\alpha} D^{n} f(t)=\frac{1}{\Gamma(n-\alpha)} \int_{0}^{t} \frac{f^{(n)}(s)}{(t-s)^{\alpha-n+1}} d s, \\
n-1<\alpha<n, n \in N, \\
f^{(n)}(t), & \alpha=n .
\end{array}\right.
$$

Defenition 1.5 [4]. Mittag-leffler functions are defined by

$$
E_{\alpha, \beta}(x)=\sum_{k=0}^{\infty} \frac{x^{k}}{\Gamma(\alpha k+\beta)}, x, \beta \in C, \operatorname{Re}(\alpha)>0, E_{\alpha}(x)=E_{\alpha, 1} .
$$

Defenition 1.6 [22]. The generalized delay exponential function (of Mittag-Leffler type) is given by

$$
G_{\alpha, \beta}^{\lambda, \tau}(t)=\sum_{j=0}^{\infty}\left(\begin{array}{l}
j+m \\
j
\end{array}\right) \frac{\lambda^{j}(t-(m+j) \tau)^{\alpha(m+j)+\beta-1}}{\Gamma(\alpha(m+j)+\beta)} H(t-(m+j) \tau), t>0,
$$


where $\lambda \in C, \alpha, \beta, \tau \in R$ and $m \in Z$ and $H(z)$ is the Heaviside step function. If $\lambda \in C, \alpha, \beta, \tau \in R$ and $m \in Z$ then laplace transform of $G_{\alpha, \beta}^{\lambda, \tau, m}(t)$ is:

$$
L\left(G_{\alpha, \beta}^{\lambda, \tau, m}(t)\right)(s)=\frac{s^{\alpha-\beta} \exp \{-m s \tau\}}{\left(s^{\alpha}-\lambda \exp \{-s \tau\}\right)^{m+1}}, \quad s>0 .
$$

\section{Governing equations}

The fundamental equations governing the unsteady motion of an incompressible fluid are

$$
\begin{gathered}
\operatorname{div} V=0, \\
\rho \frac{d V}{d t}=-\nabla p+\operatorname{div} S+F_{b} .
\end{gathered}
$$

The constitutive equation for a generalized Oldroyd-B fluid is given by [15-16],

$$
\left(1+\lambda^{\alpha} \frac{D^{\alpha}}{D t^{\alpha}}\right) S=\mu\left(1+\lambda^{\beta} \frac{D^{\beta}}{D t^{\beta}}\right) A_{1},
$$

where $V=(u, v, w)$ is the fluid velocity, $S=\left(S_{i, j}\right)$ is the extra-stress tensor, $A_{1}=(\nabla V)+(\nabla V)^{T}$ present the first Rivlin-Ericksen tensor, $\nabla$ is the gradient operator, and $p$ is the pressure. Here $F_{b}=\left(F_{b x}, F_{b y}, F_{b z}\right)$ is the body force, $\rho, \mu$ are the density and the dynamic viscosity coefficient of the fluid respectively, $\lambda_{\alpha}$ and $\lambda_{\beta}$ are the material constants that represent the relaxation time and retardation time, respectively, and $\alpha, \beta$ denote the orders of the fractional derivatives, i.e., real numbers that satisfy $0 \leq \alpha, \beta \leq 1$. Furthermore, $\frac{D^{\alpha}}{D t^{\alpha}}$ and $\frac{D^{\beta}}{D t^{\beta}}$ are fractional material derivatives that can be expressed as

$$
\begin{aligned}
& \frac{D^{\alpha} S}{D t^{\alpha}}=D_{t}^{\alpha} S+(V . \nabla) S-(\nabla \cdot V) S-S(\nabla V)^{T}, \\
& \frac{D^{\beta} S}{D t^{\beta}}=D_{t}^{\beta} S+(V . \nabla) S-(\nabla \cdot V) S-S(\nabla V)^{T} .
\end{aligned}
$$

In Eq. (3), (5), the fractional derivative operator $D^{\alpha}$ is taken in the Caputo.

We consider unidirectional flow, that is the case where the velocity and the stress take the form

$$
V=u(y, t) i, \quad S=S(y, t),
$$

where $i$ is the unit vector along the $\mathrm{x}$-direction of the Cartesian coordinate system $\mathrm{x}, \mathrm{y}$ and $\mathrm{z}$. Using Eq. (6) below, the continuity Eq.(1) is satisfied automatically while Eq. (4), bearing in mind the initial condition $S(y, 0)=0$, leads to the following relationships for the constitutive equation

$$
\begin{array}{r}
S_{x z}=S_{z y}=S_{y z}=S_{z z}=S_{y y}=0, \quad S_{y x}=S_{x y}, S_{z x}=S_{x z}, \\
\left(1+\lambda_{\alpha} D_{t}^{\alpha}\right) S_{x y}=\mu\left(1+\lambda_{\beta} D_{t}^{\beta}\right) \frac{\partial u}{\partial y}, \\
\left(1+\lambda_{\alpha} D_{t}^{\alpha}\right) S_{x x}-2 \lambda_{\alpha} S_{x y} \frac{\partial u}{\partial y}=-2 \mu \lambda_{\beta}\left(\frac{\partial u}{\partial y}\right)^{2} .
\end{array}
$$

Substituting Eqs.(6) into momentum equation (2), we have the following equation in x-direction:

$$
\left(1+\lambda_{\alpha} D_{t}^{\alpha}\right) \frac{\partial u}{\partial t}=v\left(1+\lambda_{\beta} D_{t}^{\beta}\right) \frac{\partial^{2} u}{\partial y^{2}}+\frac{1}{\rho}\left(1+\lambda_{\alpha} D_{t}^{\alpha}\right)\left(F_{b x}-\frac{\partial p}{\partial x}\right),
$$

where $\nu=\frac{\mu}{\rho}$ is the kinematic viscosity coefficient of fluid.

The constitutive equation of a generalized Burgers fluid is

$$
\left(1+\lambda_{\alpha} \frac{D^{\alpha}}{D t^{\alpha}}+\theta \frac{D^{2 \alpha}}{D t^{2 \alpha}}\right) S=\mu\left(1+\lambda_{\beta} \frac{D^{\beta}}{D t^{\beta}}\right) A_{1}, \quad(0<\alpha, \beta \leq 1),
$$

where $\theta$ is the material constant. 
Combining the constitutive equation (8) with the equation (2) we get the following fractional Burgers fluid model

$$
\left(1+\lambda_{\alpha} D_{t}^{\alpha}+\theta D_{t}^{2 \alpha}\right) \frac{\partial u}{\partial t}=v\left(1+\lambda_{\beta} D_{t}^{\beta}\right) \frac{\partial^{2} u}{\partial y^{2}}+\frac{1}{\rho}\left(1+\lambda_{\alpha} D_{t}^{\alpha}+\theta D_{t}^{2 \alpha}\right)\left(F_{b x}-\frac{\partial p}{\partial x}\right),
$$

where $\nu=\mu / \rho$. Eqs. (7)and (9) have the following form:

$$
\begin{gathered}
a_{0} D_{t}^{2 \alpha+1} u(y, t)+a_{1} D_{t}^{\alpha+1} u(y, t)+a_{2} D_{t}^{2 \alpha} u(y, t)+a_{3} D_{t}{ }^{1} u(y, t) \\
+a_{4} D_{t}^{\alpha} u(y, t)+a_{5} u(y, t)=b_{1} D_{t}^{\beta} \frac{\partial^{2} u(y, t)}{\partial y^{2}}+b_{2} \frac{\partial^{2} u(y, t)}{\partial y^{2}}+\bar{f}(y, t),
\end{gathered}
$$

the delay form of Eqs (10) is

$$
\begin{gathered}
a_{0} D_{t}^{2 \alpha+1} u(y, t)+a_{1} D_{t}^{\alpha+1} u(y, t)+a_{2} D_{t}^{2 \alpha} u(y, t)+a_{3} D_{t}{ }^{1} u(y, t) \\
+a_{4} D_{t}^{\alpha} u(y, t)+a_{5} u(y, t-\tau)=b_{1} D_{t}^{\beta} \frac{\partial^{2} u(y, t)}{\partial y^{2}}+b_{2} \frac{\partial^{2} u(y, t)}{\partial y^{2}}+\bar{f}(y, t) .
\end{gathered}
$$

The associated initial and boundary conditions are as follows:

$$
\begin{gathered}
u(y, t)=\psi_{1}(y, t), \quad u(0, t)=\varphi_{1}(t),-\tau \leq t \leq 0, \\
u_{t}(y, t)=\psi_{2}(y, t), \quad u(L, t)=\varphi_{1}(t), \quad 0<\alpha, \beta<1 .
\end{gathered}
$$

A method of separation of variables

At first, the problem involves non-homogeneous boundary conditions. We want to transform it into a problem with homogeneous boundary conditions. So, consider

$$
u(y, t)=W(y, t)+V(y, t)
$$

where

$$
V(y, t)=\left(1-\frac{y}{L}\right) \varphi_{1}(t)+\frac{y}{L} \varphi_{2}(t),
$$

which satisfies the boundary conditions

$$
V(0, t)=\varphi_{1}(t), V(L, t)=\varphi_{2}(t) .
$$

Using Eqs.(11) and Eqs.(12) along with the associated initial and boundary conditions above, we have

$$
\begin{aligned}
& W(y, t)+\left(1-\frac{y}{L}\right) \varphi_{1}(t)+\frac{y}{L} \varphi_{2}(t)=\psi_{1}(y, t), \quad-\tau \leq t \leq 0, \\
& W_{t}(y, t)+\left(1-\frac{y}{L}\right) \varphi_{1}^{\prime}(t)+\frac{y}{L} \varphi^{\prime}{ }_{2}(t)=\psi_{2}(y, t), \\
& W(L, t)+V(L, t)=\varphi_{2}(t), \\
& W(L, t)+V(L, t)=\varphi_{2}(t) \\
& W(y, t)=\psi_{1}(y, t)-\left(1-\frac{y}{L}\right) \varphi_{1}(t)-\frac{y}{L} \varphi_{2}(t)=\overline{\psi_{1}}(y, t), \\
& W_{t}(y, t)=\psi_{1}(y, t)-\left(1-\frac{y}{L}\right) \varphi_{1}^{\prime}(t)-\frac{y}{L} \varphi^{\prime}{ }_{2}(t)=\overline{\psi_{2}}(y, t) .
\end{aligned}
$$

Now main problem is solving

$$
\begin{gathered}
a_{0} D_{t}{ }^{2 \alpha+1} W(y, t)+a_{1} D_{t}{ }^{\alpha+1} W(y, t)+a_{2} D_{t}{ }^{2 \alpha} W(y, t)+a_{3} D_{t}{ }^{1} W(y, t)+ \\
\quad+a_{4} D_{t}{ }^{\alpha} W(y, t)+a_{5} W(y, t-\tau)-b_{1} D_{t}^{\beta} \frac{\partial^{2} w(y, t)}{\partial y^{2}}-b_{2} \frac{\partial^{2} w(y, t)}{\partial y^{2}}= \\
=-a_{0} D_{t}{ }^{2 \alpha+1} V(y, t)-a_{1} D_{t}^{\alpha+1} V(y, t)-a_{2} D_{t}{ }^{2 \alpha} V(y, t)-a_{3} D_{t}{ }^{1} V(y, t),
\end{gathered}
$$

where the initial condition is

$$
\sum_{n=1}^{\infty} B_{n}(0) \sin \frac{n \pi y}{L}=\sum_{n=1}^{\infty} d_{n}^{(1)}(0) \sin \frac{n \pi y}{L}-\sum_{n=1}^{\infty} \frac{2}{n \pi}\left[\varphi_{1}(0)-(-1)^{n} \varphi_{2}(0)\right] \sin \frac{n \pi y}{L},
$$




$$
\sum_{n=1}^{\infty} B^{\prime}{ }_{n}(0) \sin \frac{n \pi y}{L}=\sum_{n=1}^{\infty} d_{n}^{(2)}(0) \sin \frac{n \pi y}{L}-\sum_{n=1}^{\infty} \frac{2}{n \pi}\left[\varphi^{\prime}{ }_{1}(0)-(-1)^{n} \varphi_{2}^{\prime}(0)\right] \sin \frac{n \pi y}{L}
$$

and

$$
d_{n}^{(i)}=\frac{2}{L} \int_{0}^{L} \bar{\psi}_{i}(y, 0) \sin \frac{n \pi y}{L} d y, \quad i=1,2 .
$$

Let

$$
\begin{gathered}
W(y, t)=\sum_{n=1}^{\infty} B_{n}(t) \sin \frac{n \pi y}{L} \\
\overline{\psi_{i}}(y)=\sum_{n=1}^{\infty} d_{n}^{(i)} \sin \frac{n \pi y}{L} \quad(i=1,2, \ldots, m) .
\end{gathered}
$$

Then, we have

$$
\begin{gathered}
a_{0} D_{t}{ }^{2 \alpha+1} \sum_{n=1}^{\infty} B_{n}(t) \sin \frac{n \pi y}{L}+a_{1} D_{t}{ }^{\alpha+1} \sum_{n=1}^{\infty} B_{n}(t) \sin \frac{n \pi y}{L}+a_{2} D_{t}^{2 \alpha} \sum_{n=1}^{\infty} B_{n}(t) \sin \frac{n \pi y}{L}+ \\
+a_{3} D_{t}{ }^{1} \sum_{n=1}^{\infty} B_{n}(t) \sin \frac{n \pi y}{L}+a_{4} D_{t}{ }^{\alpha} \sum_{n=1}^{\infty} B_{n}(t) \sin \frac{n \pi y}{L}+a_{5} \sum_{n=1}^{\infty} B_{n}(t-\tau) \sin \frac{n \pi y}{L}- \\
-b_{1}\left(\frac{n \pi}{L}\right)^{2} D_{t}^{\beta} \sum_{n=1}^{\infty} B_{n}(t) \sin \frac{n \pi y}{L}-b_{2}\left(\frac{n \pi}{L}\right)^{2} \sum_{n=1}^{\infty} B_{n}(t) \sin \frac{n \pi y}{L}= \\
=-a_{0} \frac{2}{n \pi} D_{t}{ }^{2 \alpha+1} \sum_{n=1}^{\infty}\left[\varphi_{1}(t)-(-1)^{n} \varphi_{2}(t)\right] \sin \frac{n \pi y}{L}-a_{1} \frac{2}{n \pi} D_{t}^{\alpha+1} \sum_{n=1}^{\infty}\left[\varphi_{1}(t)-(-1)^{n} \varphi_{2}(t)\right] \sin \frac{n \pi y}{L}- \\
-a_{2} \frac{2}{n \pi} D_{t}{ }^{2 \alpha} \sum_{n=1}^{\infty}\left[\varphi_{1}(t)-(-1)^{n} \varphi_{2}(t)\right] \sin \frac{n \pi y}{L}-a_{3} \frac{2}{n \pi} D_{t}{ }^{1} \sum_{n=1}^{\infty}\left[\varphi_{1}(t)-(-1)^{n} \varphi_{2}(t)\right] \sin \frac{n \pi y}{L}- \\
-a_{4} \frac{2}{n \pi} D_{t}{ }^{\alpha} \sum_{n=1}^{\infty}\left[\varphi_{1}(t)-(-1)^{n} \varphi_{2}(t)\right] \sin \frac{n \pi y}{L}-a_{5} \frac{2}{n \pi} \sum_{n=1}^{\infty}\left[\varphi_{1}(t-\tau)-(-1)^{n} \varphi_{2}(t-\tau)\right] \sin \frac{n \pi y}{L}+ \\
+\sum_{n=1}^{\infty} f_{n}(t) \sin \frac{n \pi y}{L} .
\end{gathered}
$$

Equating coefficients leads to

$$
\begin{gathered}
a_{0} D_{t}{ }^{2 \alpha+1} B_{n}(t)+a_{1} D_{t}^{\alpha+1} B_{n}(t)+a_{2} D_{t}{ }^{2 \alpha} B_{n}(t)+a_{3} D_{t}{ }^{1} B_{n}(t)+ \\
+a_{4} D_{t}{ }^{\alpha} B_{n}(t)+a_{5} B_{n}(t-\tau)-b_{1}\left(\frac{n \pi}{L}\right)^{2} D_{t}^{\beta} B_{n}(t)-b_{2}\left(\frac{n \pi}{L}\right)^{2} B_{n}(t)= \\
=-a_{0} \frac{2}{n \pi} D_{t}{ }^{2 \alpha+1}\left[\varphi_{1}(t)-(-1)^{n} \varphi_{2}(t)\right]-a_{1} \frac{2}{n \pi} D_{t}^{\alpha+1}\left[\varphi_{1}(t)-(-1)^{n} \varphi_{2}(t)\right]- \\
-a_{2} \frac{2}{n \pi} D_{t}{ }^{2 \alpha}\left[\varphi_{1}(t)-(-1)^{n} \varphi_{2}(t)\right]-a_{3} \frac{2}{n \pi} D_{t}{ }^{1}\left[\varphi_{1}(t)-(-1)^{n} \varphi_{2}(t)\right]- \\
-a_{4} \frac{2}{n \pi} D_{t}^{\alpha}\left[\varphi_{1}(t)-(-1)^{n} \varphi_{2}(t)\right]-a_{5} \frac{2}{n \pi}\left[\varphi_{1}(t-\tau)-(-1)^{n} \varphi_{2}(t-\tau)\right]+f_{n}(t),
\end{gathered}
$$

with the boundary conditions

$$
\begin{aligned}
B_{n}(0) & =d_{n}^{(1)}(0)-\frac{2}{n \pi} \varphi_{1}(0)+(-1)^{n} \frac{2}{n \pi} \varphi_{2}(0), \\
B_{n}^{\prime}(0) & =d_{n}^{(2)}(0)-\frac{2}{n \pi} \varphi_{1}^{\prime}(0)+(-1)^{n} \frac{2}{n \pi} \varphi_{2}^{\prime}(0) .
\end{aligned}
$$

In this part we divide the main problem in two part 


$$
3.1\left(0 \leq \alpha, \beta \leq \frac{1}{2}\right)
$$

when $\frac{1}{2} \leq \alpha, \beta \leq 1$ and applying the Laplace transform with respect to $\mathrm{t}$ defined by

$$
\bar{B}_{n}(s)=\int_{0}^{\infty} e^{-s t} B_{n}(t) d t
$$

In Eq.(13), we obtain

$$
\begin{gathered}
a_{0} s^{2 \alpha+1} \bar{B}_{n}(s)-a_{0} s^{2 \alpha} B_{n}(0)+a_{1} s^{\alpha+1} \bar{B}_{n}(s)-a_{1} s^{\alpha} B_{n}(0)+a_{2} s^{2 \alpha} \bar{B}_{n}(s)-a_{2} s^{2 \alpha-1} B_{n}(0)+ \\
\left.+a_{3} s \bar{B}_{n}(s)-a_{3} B_{n}(0)+a_{4} s^{\alpha} \bar{B}_{n}(s)-a_{4} s^{\alpha-1} B_{n}(0)+a_{5} e^{-s \tau} e^{-s p} B_{n}(p) d p\right]- \\
-a_{5} e^{-s \tau} \bar{B}_{n}(s)-b_{1}\left(\frac{n \pi}{L}\right)^{2} s^{\beta} \bar{B}_{n}(s)+b_{1}\left(\frac{n \pi}{L}\right)^{2} s^{\beta-1} B_{n}(0)-b_{2}\left(\frac{n \pi}{L}\right)^{2} \bar{B}_{n}(s)= \\
=-a_{0} \frac{2}{n \pi} s^{2 \alpha+1}\left[\overline{\varphi_{1}}(s)-(-1)^{n} \overline{\varphi_{2}}(s)\right]+a_{0} \frac{2}{n \pi} s^{2 \alpha}\left[d_{n}^{(1)}(0)-B_{n}(0)\right]- \\
-a_{1} \frac{2}{n \pi} s^{\alpha+1}\left[\overline{\varphi_{1}}(s)-(-1)^{n} \overline{\varphi_{2}}(s)\right]+a_{1} \frac{2}{n \pi} s^{\alpha}\left[d_{n}^{(1)}(0)-B_{n}(0)\right]- \\
-a_{2} \frac{2}{n \pi} s^{2 \alpha}\left[\overline{\varphi_{1}}(s)-(-1)^{n} \overline{\varphi_{2}}(s)\right]+a_{2} \frac{2}{n \pi} s^{2 \alpha-1}\left[d_{n}^{(1)}(0)-B_{n}(0)\right]- \\
-a_{3} \frac{2}{n \pi} s\left[\overline{\varphi_{1}}(s)-(-1)^{n} \overline{\varphi_{2}}(s)\right]+a_{3} \frac{2}{n \pi}\left[d_{n}^{(1)}(0)-B_{n}(0)\right]- \\
-a_{4} \frac{2}{n \pi} s^{\alpha}\left[\overline{\varphi_{1}}(s)-(-1)^{n} \overline{\varphi_{2}}(s)\right]+a_{4} \frac{2}{n \pi} s^{\alpha-1}\left[d_{n}^{(1)}(0)-B_{n}(0)\right]-a_{5} \frac{2}{n \pi} e^{-s \tau}\left[\overline{\varphi_{1}}(s)-(-1)^{n} \overline{\varphi_{2}}(s)\right]+ \\
+a_{5} \frac{2}{n \pi} e^{-s \tau}\left[\int_{-\tau}^{0} e^{-s p}\left[\varphi_{1}(p)-(-1)^{n} \varphi_{2}(p)\right] d p\right]+F_{n}(s) . \\
0
\end{gathered}
$$

By assumption $H(S)=\int_{-\tau}^{0} e^{-s p}\left[\varphi_{1}(p)-(-1)^{n} \varphi_{2}(p)\right] d p, \quad G(s)=\int_{-\tau}^{0} e^{-s p} B_{n}(p) d p$ and $k_{n}=\frac{n \pi}{L}$, so we can write

$$
\begin{gathered}
\bar{B}_{n}(s)=\frac{B_{n}(0)\left[a_{0} s^{2 \alpha}+a_{1} s^{\alpha}+a_{2} s^{2 \alpha-1}+a_{3}+a_{4} s^{\alpha-1}-b_{1} k_{n}{ }^{2} s^{\beta-1}\right]}{a_{0} s^{2 \alpha+1}+a_{1} s^{\alpha+1}+a_{2} s^{2 \alpha}+a_{3} s+a_{4} s^{\alpha}-a_{5} e^{-s \tau}-b_{1} k_{n}{ }^{2} s^{\beta}-b_{2} k_{n}{ }^{2}}+ \\
+\frac{-\frac{2}{k_{n} L}\left[\overline{\varphi_{1}}(s)-(-1)^{n} \overline{\varphi_{2}}(s)\right]\left[a_{0} s^{2 \alpha+1}+a_{1} s^{\alpha+1}+a_{2} s^{2 \alpha}+a_{3} s+a_{4} s^{\alpha}+a_{5} e^{-s \tau}\right]}{a_{0} s^{2 \alpha+1}+a_{1} s^{\alpha+1}+a_{2} s^{2 \alpha}+a_{3} s+a_{4} s^{\alpha}-a_{5} e^{-s \tau}-b_{1} k_{n}{ }^{2} s^{\beta}-b_{2} k_{n}{ }^{2}}+ \\
+\frac{\frac{2}{k_{n} L}\left[d_{n}^{(1)}(0)-B_{n}(0)\right]\left\{a_{0} s^{2 \alpha}+a_{1} s^{\alpha}+a_{2} s^{2 \alpha-1}+a_{3}+a_{4} s^{\alpha-1}\right\}}{a_{0} s^{2 \alpha+1}+a_{1} s^{\alpha+1}+a_{2} s^{2 \alpha}+a_{3} s+a_{4} s^{\alpha}-a_{5} e^{-s \tau}-b_{1} k_{n}{ }^{2} s^{\beta}-b_{2} k_{n}{ }^{2}}+ \\
+\frac{-a_{5} G(s) e^{-s \tau}+a_{5} \frac{2}{k_{n} L} e^{-s \tau} H(S)+F_{n}(s)}{a_{0} s^{2 \alpha+1}+a_{1} s^{\alpha+1}+a_{2} s^{2 \alpha}+a_{3} s+a_{4} s^{\alpha}-a_{5} e^{-s \tau}-b_{1} k_{n}{ }^{2} s^{\beta}-b_{2} k_{n}{ }^{2}} .
\end{gathered}
$$

Using Eq.(14) we rewrite Eq.(13) as

$$
\bar{B}_{n}(s)=e^{s m \tau} \sum_{m=0}^{\infty} \sum_{k, i, j, l, n, q \geq 0}^{k+i+j+l+n+q=m} \frac{(-1)^{m}}{a_{0} m+1} \frac{m !\left(-k_{n}{ }^{2}\right)^{n+q}}{k ! i ! j ! l ! q !} a_{1}{ }^{k} a_{2}{ }^{i} a_{3}{ }^{j} a_{4}{ }^{l} b_{1}{ }^{n} b_{2}{ }^{q}
$$




$$
\begin{aligned}
& \left\{B _ { n } ( 0 ) \left[a_{0} \frac{s^{\alpha(k+2 i+l+2)+k+\beta n} e^{-s m \tau}}{\left(s^{2 \alpha+1}-\frac{a_{5}}{a_{0}} e^{-s \tau}\right)^{m+1}}+a_{1} \frac{s^{\alpha(k+2 i+l+1)+k+\beta n} e^{-s m \tau}}{\left(s^{2 \alpha+1}-\frac{a_{5}}{a_{0}} e^{-s \tau}\right)^{m+1}}+\right.\right. \\
& +a_{2} \frac{s^{\alpha(k+2 i+l+2)+k+\beta n-1} e^{-s m \tau}}{\left(s^{2 \alpha+1}-\frac{a_{5}}{a_{0}} e^{-s \tau}\right)^{m+1}}+a_{3} \frac{s^{\alpha(k+2 i+l)+k+\beta n} e^{-s m \tau}}{\left(s^{2 \alpha+1}-\frac{a_{5}}{a_{0}} e^{-s \tau}\right)^{m+1}}+ \\
& \left.+a_{4} \frac{s^{\alpha(k+2 i+l+1)+k+\beta n} e^{-s m \tau}}{\left(s^{2 \alpha+1}-\frac{a_{5}}{a_{0}} e^{-s \tau}\right)^{m+1}}+a_{5} e^{-s \tau} \frac{s^{\alpha(k+2 i+l)+k+\beta n} e^{-s m \tau}}{\left(s^{2 \alpha+1}-\frac{a_{5}}{a_{0}} e^{-s \tau}\right)^{m+1}}\right]- \\
& -\frac{2}{k_{n} L}\left[\overline{\varphi_{1}}(s)-(-1)^{n} \overline{\varphi_{2}}(s)\right]\left[a_{0} \frac{s^{\alpha(k+2 i+l+2)+k+\beta n+1} e^{-s m \tau}}{\left(s^{2 \alpha+1}-\frac{a_{5}}{a_{0}} e^{-s \tau}\right)^{m+1}}+a_{1} \frac{s^{\alpha(k+2 i+l+1)+k+\beta n+1} e^{-s m \tau}}{\left(s^{2 \alpha+1}-\frac{a_{5}}{a_{0}} e^{-s \tau}\right)^{m+1}}+\right. \\
& +a_{2} \frac{s^{\alpha(k+2 i+l+2)+k+\beta n} e^{-s m \tau}}{\left(s^{2 \alpha+1}-\frac{a_{5}}{a_{0}} e^{-s \tau}\right)^{m+1}}+a_{3} \frac{s^{\alpha(k+2 i+l)+k+\beta n+1} e^{-s m \tau}}{\left(s^{2 \alpha+1}-\frac{a_{5}}{a_{0}} e^{-s \tau}\right)^{m+1}}+ \\
& \left.+a_{4} \frac{s^{\alpha(k+2 i+l+1)+k+\beta n} e^{-s m \tau}}{\left(s^{2 \alpha+1}-\frac{a_{5}}{a_{0}} e^{-s \tau}\right)^{m+1}}+a_{5} e^{-s \tau} \frac{s^{\alpha(k+2 i+l)+k+\beta n} e^{-s m \tau}}{\left(s^{2 \alpha+1}-\frac{a_{5}}{a_{0}} e^{-s \tau}\right)^{m+1}}\right]+ \\
& +\frac{2}{k_{n} L}\left[d_{n}^{(1)}(0)-B_{n}(0)\right]\left[a_{0} \frac{s^{\alpha(k+2 i+l+2)+k+\beta n} e^{-s m \tau}}{\left(s^{2 \alpha+1}-\frac{a_{5}}{a_{0}} e^{-s \tau}\right)^{m+1}}+a_{1} \frac{s^{\alpha(k+2 i+l+1)+k+\beta n} e^{-s m \tau}}{\left(s^{2 \alpha+1}-\frac{a_{5}}{a_{0}} e^{-s \tau}\right)^{m+1}}+\right. \\
& \left.+a_{2} \frac{s^{\alpha(k+2 i+l+2)+k+\beta n-1} e^{-s m \tau}}{\left(s^{2 \alpha+1}-\frac{a_{5}}{a_{0}} e^{-s \tau}\right)^{m+1}}+a_{3} \frac{s^{\alpha(k+2 i+l)+k+\beta n} e^{-s m \tau}}{\left(s^{2 \alpha+1}-\frac{a_{5}}{a_{0}} e^{-s \tau}\right)^{m+1}}+a_{4} \frac{s^{\alpha(k+2 i+l+1)+k+\beta n-1} e^{-s m \tau}}{\left(s^{2 \alpha+1}-\frac{a_{5}}{a_{0}} e^{-s \tau}\right)^{m+1}}\right]- \\
& -a_{5} G(s) e^{-s \tau} \frac{s^{\alpha(k+2 i+l)+k+\beta n} e^{-s m \tau}}{\left(s^{2 \alpha+1}-\frac{a_{5}}{a_{0}} e^{-s \tau}\right)^{m+1}}+a_{5} \frac{2}{k_{n} L} e^{-s \tau} H(S) \frac{s^{\alpha(k+2 i+l)+k+\beta n} e^{-s m \tau}}{\left(s^{2 \alpha+1}-\frac{a_{5}}{a_{0}} e^{-s \tau}\right)^{m+1}}+ \\
& +F_{n}(s) \frac{s^{\alpha(k+2 i+l)+k+\beta n} e^{-s m \tau}}{\left(s^{2 \alpha+1}-\frac{a_{5}}{a_{0}} e^{-s \tau}\right)^{m+1}} .
\end{aligned}
$$

Applying the discrete inverse Laplace transform to the preceding equation, we obtain

$$
B_{n}(t)=\sum_{m=0}^{\infty} \sum_{k, i, j, l, n, q \geq 0}^{k+i+j+l+n+q=m} \frac{(-1)^{m}}{a_{0}{ }^{m+1}} \frac{m !\left(-k_{n}{ }^{2}\right)^{n+q}}{k ! i ! j ! l ! q !} a_{1}{ }^{k} a_{2}{ }^{i} a_{3}{ }^{j} a_{4}{ }^{l} b_{1}{ }^{n} b_{2}{ }^{q}
$$

$$
\left\{B _ { n } ( 0 ) H ( t - m \tau ) \left[a_{0} G_{2 \alpha+1,-\alpha(k+2 i+l)-k-\beta n+1}^{\left(\frac{a_{5}}{a_{0}}\right), \tau, m}(t-m \tau)+a_{1} G_{2 \alpha+1,-\alpha(k+2 i+l-1)-k-\beta n+1}^{\left(\frac{a_{5}}{a_{0}}\right), \tau, m}(t-m \tau)+\right.\right.
$$$$
+a_{2} G_{2 \alpha+1,-\alpha(k+2 i+l)-k-\beta n+2}^{\left(\frac{a_{5}}{a_{0}}\right), \tau, m}(t-m \tau)+a_{3} G_{2 \alpha+1,-\alpha(k+2 i+l-2)-k-\beta n+1}^{\left(\frac{a_{5}}{a_{0}}\right), \tau, m}(t-m \tau)+
$$$$
\left.+a_{4} G_{2 \alpha+1,-\alpha(k+2 i+l-1)-k-\beta n+2}^{\left(\frac{a_{5}}{a_{0}}\right), \tau, m}(t-m \tau)-b_{1} k_{n}{ }^{2} G_{2 \alpha+1,-\alpha(k+2 i+l-2)-k-\beta n+2}^{\left(\frac{a_{5}}{a_{0}}\right), \tau, m}(t-m \tau)\right]-
$$$$
-\frac{2}{k_{n} L}\left[\int _ { 0 } ^ { t } [ \varphi _ { 1 } ( t - u ) - ( - 1 ) ^ { n } \varphi _ { 2 } ( t - u ) ] H ( u - m \tau ) \left(a_{0} G_{2 \alpha+1,-\alpha(k+2 i+l)-k-\beta n}^{\left(\frac{a_{5}}{a_{0}}\right), \tau, m}(u-m \tau)+\right.\right.
$$

$$
\begin{gathered}
+a_{1} G_{2 \alpha+1,-\alpha(k+2 i+l-1)-k-\beta n}^{\left(\frac{a_{5}}{a_{0}}\right), \tau, m}(u-m \tau)+a_{2} G_{2 \alpha+1,-\alpha(k+2 i+l)-k-\beta n+1}^{\left(\frac{a_{5}}{a_{0}}\right), \tau, m}(u-m \tau)+ \\
\left.\left.+a_{3} G_{2 \alpha+1,-\alpha(k+2 i+l-2)-k-\beta n}^{\left(\frac{a_{5}}{a_{0}}\right), \tau, m}(u-m \tau)+a_{4} G_{2 \alpha+1,-\alpha(k+2 i+l-1)-k-\beta n+1}^{\left(\frac{a_{5}}{a_{0}}\right), \tau, m}(u-m \tau)\right) d u\right]-
\end{gathered}
$$




$$
\begin{gathered}
-a_{5} \frac{2}{k_{n} L} \int_{0}^{t}\left[\varphi_{1}(t-u)-(-1)^{n} \varphi_{2}(t-u)\right] H(u-\tau(m+1)) \\
G_{2 \alpha+1,-\alpha(k+2 i+l-2)-k-\beta n+1}^{\left(\frac{a_{5}}{a_{0}}\right), \tau, m}(u-\tau(m+1)) d u+ \\
+\frac{2}{k_{n} L}\left[d_{n}^{(1)}(0)-B_{n}(0)\right]\left[a_{0} G_{2 \alpha+1,-\alpha(k+2 i+l)-k-\beta n+1}^{\left(\frac{a_{5}}{a_{0}}\right), \tau, m}(t-m \tau)+\right. \\
+a_{1} G_{2 \alpha+1,-\alpha(k+2 i+l-1)-k-\beta n+1}^{\left(\frac{a_{5}}{a_{0}}\right), \tau, m}(t-m \tau)+a_{2} G_{2 \alpha+1,-\alpha(k+2 i+l)-k-\beta n+2}^{\left(\frac{a_{5}}{a_{0}}\right), \tau, m}(t-m \tau)+ \\
\left.+a_{3} G_{2 \alpha+1,-\alpha(k+2 i+l-2)-k-\beta n+1}^{\left(\frac{a_{5}}{a_{0}}\right), \tau, m}(t-m \tau)+a_{4} G_{2 \alpha+1,-\alpha(k+2 i+l-1)-k-\beta n+2}^{\left(\frac{a_{5}}{a_{0}}\right), \tau, m}(t-m \tau)\right]- \\
-a_{5} \int_{0}^{t} g(t-u) H(u-\tau(m+1)) G_{2 \alpha+1,-\alpha(k+2 i+l-2)-k-\beta n+1}^{\left(\frac{a_{5}}{a_{0}}\right), \tau, m}(u-\tau(m+1)) d u- \\
-a_{5} \frac{2}{k_{n} L} \int_{0}^{t} h(t-u) H(u-\tau(m+1)) G_{2 \alpha+1,-\alpha(k+2 i+l-2)-k-\beta n+1}^{\left(\frac{a_{5}}{a_{0}}\right), \tau, m}(u-\tau(m+1)) d u+ \\
+\int_{0}^{t} f_{n}(t-u) H(u-m \tau) G_{2 \alpha+1,-\alpha(k+2 i+l-2)-k-\beta n+1}^{\left(\frac{a_{5}}{a_{0}}\right), \tau, m}(u-\tau(m+1)) d u
\end{gathered}
$$

Once the $B_{n}(t)$ are known, so are the $W(y, t)$, and thus $u(y, t)$ as desired.

$$
3.2\left(\frac{1}{2} \leq \alpha, \beta \leq 1\right)
$$

In the same way in the subsection 3.1 we could have

$$
B_{n}(t)=\sum_{m=0}^{\infty} \sum_{k, i, j, l, n, q \geq 0}^{k+i+j+l+n+q=m} \frac{(-1)^{m}}{a_{0}{ }^{m+1}} \frac{m !\left(-k_{n}{ }^{2}\right)^{n+q}}{k ! i ! j ! l ! q !} a_{1}{ }^{k} a_{2}{ }^{i} a_{3}{ }^{j} a_{4}{ }^{l} b_{1}{ }^{n} b_{2}{ }^{q}
$$

$\left\{B_{n}(0) H(t-m \tau)\left[a_{0} G_{2 \alpha+1,-\alpha(k+2 i+l)-k-\beta n+1}^{\left(\frac{a_{5}}{a_{0}}\right), \tau, m}(t-m \tau)+a_{1} G_{2 \alpha+1,-\alpha(k+2 i+l-1)-k-\beta n+1}^{\left(\frac{a_{5}}{a_{0}}\right), \tau, m}(t-m \tau)+\right.\right.$

$$
\begin{aligned}
& +a_{2} G_{2 \alpha+1,-\alpha(k+2 i+l)-k-\beta n+2}^{\left(\frac{a_{5}}{a_{0}}\right), \tau, m}(t-m \tau)+a_{3} G_{2 \alpha+1,-\alpha(k+2 i+l-2)-k-\beta n+1}^{\left(\frac{a_{5}}{a_{0}}\right), \tau, m}(t-m \tau)+ \\
+ & \left.a_{4} G_{2 \alpha+1,-\alpha(k+2 i+l-1)-k-\beta n+2}^{\left(\frac{a_{5}}{a_{0}}\right), \tau, m}(t-m \tau)-b_{1} k_{n}{ }^{2} G_{2 \alpha+1,-\alpha(k+2 i+l-2)-k-\beta n+2}^{\left(\frac{a_{5}}{a_{0}}\right), \tau, m}(t-m \tau)\right]+
\end{aligned}
$$

$+B^{\prime}{ }_{n}(0) H(t-m \tau)\left[a_{0} G_{2 \alpha+1,-\alpha(k+2 i+l)-k-\beta n+2}^{\left(\frac{a_{5}}{a_{0}}\right), \tau, m}(t-m \tau)+a_{1} G_{2 \alpha+1,-\alpha(k+2 i+l-1)-k-\beta n+2}^{\left(\frac{a_{5}}{a_{0}}\right), \tau, m}(t-m \tau)+\right.$

$$
\begin{aligned}
& \left.+a_{2} G_{2 \alpha+1,-\alpha(k+2 i+l)-k-\beta n+3}^{\left(\frac{a_{5}}{a_{0}}\right), \tau, m}(t-m \tau)+a_{4} G_{2 \alpha+1,-\alpha(k+2 i+l-1)-k-\beta n+3}^{\left(\frac{a_{5}}{a_{0}}\right), \tau, m}(t-m \tau)\right]+ \\
& \quad+B_{n}^{\prime \prime}(0) a_{0} G_{2 \alpha+1,-\alpha(k+2 i+l)-k-\beta n+3}^{\left(\frac{a_{5}}{a_{0}}\right), \tau, m}(t-m \tau)- \\
& -\frac{2}{k_{n} L}\left[\int _ { 0 } ^ { t } [ \varphi _ { 1 } ( t - u ) - ( - 1 ) ^ { n } \varphi _ { 2 } ( t - u ) ] H ( u - m \tau ) \left(a_{0} G_{2 \alpha+1,-\alpha(k+2 i+l)-k-\beta n}^{\left(\frac{a_{5}}{a_{0}}\right), \tau, m}(u-m \tau)+\right.\right. \\
& \quad+a_{1} G_{2 \alpha+1,-\alpha(k+2 i+l-1)-k-\beta n}^{\left(\frac{a_{5}}{a_{0}}\right), \tau, m}(u-m \tau)+a_{2} G_{2 \alpha+1,-\alpha(k+2 i+l)-k-\beta n+1}^{\left(\frac{a_{5}}{a_{0}}\right), \tau, m}(u-m \tau)+ \\
& \left.\left.+a_{3} G_{2 \alpha+1,-\alpha(k+2 i+l-2)-k-\beta n}^{\left(\frac{a_{5}}{a_{0}}\right), \tau, m}(u-m \tau)+a_{4} G_{2 \alpha+1,-\alpha(k+2 i+l-1)-k-\beta n+1}^{\left(\frac{a_{5}}{a_{0}}\right), \tau, m}(u-m \tau)\right) d u\right]-
\end{aligned}
$$




$$
\begin{aligned}
& -a_{5} \frac{2}{k_{n} L} \int_{0}^{t}\left[\varphi_{1}(t-u)-(-1)^{n} \varphi_{2}(t-u)\right] H(u-\tau(m+1)) G_{2 \alpha+1,-\alpha(k+2 i+l-2)-k-\beta n+1}^{\left(\frac{a_{5}}{a_{0}}\right), \tau, m}(u-\tau(m+1)) d u+ \\
& +\frac{2}{k_{n} L}\left[d_{n}^{(1)}(0)-B_{n}(0)\right]\left[a_{0} G_{2 \alpha+1,-\alpha(k+2 i+l)-k-\beta n+2}^{\left(\frac{a_{5}}{a_{0}}\right), \tau, m}(t-m \tau)+\right. \\
& \left.+a_{1} G_{2 \alpha+1,-\alpha(k+2 i+l-1)-k-\beta n+2}^{\left(\frac{a_{5}}{a_{0}}\right), \tau, m}(t-m \tau)+a_{2} G_{2 \alpha+1,-\alpha(k+2 i+l)-k-\beta n+3}^{\left(\frac{a_{5}}{a_{0}}\right), \tau, m}(t-m \tau)\right]+ \\
& +\frac{2}{k_{n} L}\left[d_{n}^{(2)}(0)-B_{n}^{\prime}(0)\right]\left[a_{0} G_{2 \alpha+1,-\alpha(k+2 i+l)-k-\beta n+2}^{\left(\frac{a_{5}}{a_{0}}\right), \tau, m}(t-m \tau)+\right. \\
& \left.+a_{1} G_{2 \alpha+1,-\alpha(k+2 i+l-1)-k-\beta n+2}^{\left(\frac{a_{5}}{a_{0}}\right), \tau, m}(t-m \tau)+a_{2} G_{2 \alpha+1,-\alpha(k+2 i+l)-k-\beta n+3}^{\left(\frac{a_{5}}{a_{0}}\right), \tau, m}(t-m \tau)\right]+ \\
& +\frac{2}{k_{n} L}\left[d_{n}^{(3)}(0)-B_{n}^{\prime \prime}(0)\right] a_{0} G_{2 \alpha+1,-\alpha(k+2 i+l)-k-\beta n+3}^{\left(\frac{a_{5}}{a_{0}}\right), \tau, m}(t-m \tau)- \\
& -a_{5} \int_{0}^{t} g(t-u) H(u-\tau(m+1)) G_{2 \alpha+1,-\alpha(k+2 i+l-2)-k-\beta n+1}^{\left(\frac{a_{5}}{a_{0}}\right), \tau, m}(u-\tau(m+1)) d u- \\
& -a_{5} \frac{2}{k_{n} L} \int_{0}^{t} h(t-u) H(u-\tau(m+1)) G_{2 \alpha+1,-\alpha(k+2 i+l-2)-k-\beta n+1}^{\left(\frac{a_{5}}{a_{0}}\right), \tau, m}(u-\tau(m+1)) d u+ \\
& \left.+\int_{0}^{t} f_{n}(t-u) H(u-m \tau) G_{2 \alpha+1,-\alpha(k+2 i+l-2)-k-\beta n+1}^{\left(\frac{a_{5}}{a_{0}}\right), \tau, m}(u-\tau(m+1)) d u\right\} .
\end{aligned}
$$

\section{Examples}

We consider the flow of an Oldroyd-B fluid when the body force and the pressure gradient are omitted and the plate is accelerating. We present the analytical solution in the different initial conditions

Example 1. In this example the plate is moving at speed $c t$, where $c$ is constant. The corresponding initial problem is then given as

$$
\begin{gathered}
\frac{\partial u(y, t)}{\partial t}+\lambda_{\alpha} D_{t}^{\alpha} u(y, t)+\theta D_{t}^{2 \alpha} u(y, t)=\nu \frac{\partial^{2} u(y, t)}{\partial y^{2}}+\nu \lambda_{\beta} D_{t}^{\beta} \frac{\partial^{2} u(y, t)}{\partial y^{2}}-M u(y, t-\tau) \\
u(y, t)=c_{1}, \quad u(0, t)=c t, \quad-\tau \leq t \leq 0, \quad y>0, \\
u_{t}(y, t)=c_{2}, \quad u(L, t)=0, \quad \frac{1}{2}<\alpha, \beta<1 \\
u_{t t}(y, t)=0 .
\end{gathered}
$$

Separating variables and use of the Laplace transformation yields,

$$
\begin{aligned}
& \bar{B}_{n}(s)=e^{s m \tau} \sum_{m=0}^{\infty} \sum_{k, i, j \geq 0}^{k+i+j=m} \frac{(-1)^{m}}{(\theta)^{m+1}} \frac{m !\left(-k_{n}{ }^{2} \nu\right)^{j+l} \lambda_{\beta}{ }^{l} \lambda_{\alpha}{ }^{i}}{k ! i ! j ! l !} \\
& \left\{B _ { n } ( 0 ) \left[\frac{s^{k+\alpha i+\beta l} e^{-s m \tau}}{\left(s^{2 \alpha}-\frac{M}{\theta} e^{-s \tau}\right)^{m+1}}+\lambda_{\alpha} \frac{s^{k+\alpha(i+1)+\beta l-1} e^{-s m \tau}}{\left(s^{2 \alpha}-\frac{M}{\theta} e^{-s \tau}\right)^{m+1}}+\theta \frac{s^{k+\alpha(i+2)+\beta l-1} e^{-s m \tau}}{\left(s^{2 \alpha}-\frac{M}{\theta} e^{-s \tau}\right)^{m+1}}\right.\right. \\
& \left.-\nu \lambda_{\beta} k_{n}{ }^{2} \frac{s^{k+\alpha i+\beta(1+l)-1} e^{-s m \tau}}{\left(s^{2 \alpha}-\frac{M}{\theta} e^{-s \tau}\right)^{m+1}}\right]+B^{\prime}{ }_{n}(0)\left[\lambda_{\alpha} \frac{s^{k+\alpha(1+i)+\beta l-2} e^{-s m \tau}}{\left(s^{2 \alpha}-\frac{M}{\theta} e^{-s \tau}\right)^{m+1}}+\theta \frac{s^{k+\alpha(i+2)+\beta l-2} e^{-s m \tau}}{\left(s^{2 \alpha}-\frac{M}{\theta} e^{-s \tau}\right)^{m+1}}\right. \\
& \left.+\nu \lambda_{\beta} k_{n}{ }^{2} \frac{s^{k+\alpha i+\beta(1+l)-2} e^{-s m \tau}}{\left(s^{2 \alpha}-\frac{M}{\theta} e^{-s \tau}\right)^{m+1}}\right]-M e^{-s \tau} G(s) \frac{s^{k+\alpha i+\beta l} e^{-s m \tau}}{\left(s^{2 \alpha}-\frac{M}{\theta} e^{-s \tau}\right)^{m+1}} \\
& +\frac{2 c}{k_{n} L}\left[\frac{s^{k+\alpha i+\beta l-1} e^{-s m \tau}}{\left(s^{2 \alpha}-\frac{M}{\theta} e^{-s \tau}\right)^{m+1}}-M e^{-s \tau} \frac{s^{k+\alpha i+\beta l-2} e^{-s m \tau}}{\left(s^{2 \alpha}-\frac{M}{\theta} e^{-s \tau}\right)^{m+1}}+M e^{-s \tau} H(s) \frac{s^{k+\alpha i+\beta l} e^{-s m \tau}}{\left(s^{2 \alpha}-\frac{M}{\theta} e^{-s \tau}\right)^{m+1}}-\lambda_{\alpha} \frac{s^{k+\alpha(1+i)+\beta l-2} e^{-s m \tau}}{\left(s^{2 \alpha}-\frac{M}{\theta} e^{-s \tau}\right)^{m+1}}\right. \\
& \left.\left.-\theta \Gamma(-\alpha+2) \frac{s^{k+\alpha(2+i)+\beta l-2} e^{-s m \tau}}{\left(s^{2 \alpha}-\frac{M}{\theta} e^{-s \tau}\right)^{m+1}}\right]\right\} .
\end{aligned}
$$


Taking inverse Laplace transform gives us

$B_{n}(t)=\sum_{m=0}^{\infty} \sum_{k, i, j \geq 0}^{k+i+j=m} \frac{(-1)^{m}}{(\theta)^{m+1}} \frac{m !\left(-k_{n}{ }^{2} \nu\right)^{j+l} \lambda_{\beta}{ }^{l} \lambda_{\alpha}{ }^{i}}{k ! i ! j ! ! !}$

$\left\{B_{n}(0) H(t-m \tau)\left[G_{2 \alpha,-k-\alpha(i-2)-\beta l}^{\left(\frac{M}{\theta}\right), \tau, m}(t-m \tau)+\lambda_{\alpha} G_{2 \alpha,-k-\alpha(i-1)-\beta l+1}^{\left(\frac{M}{\theta}\right), \tau, m}(t-m \tau)+\theta G_{2 \alpha,-k-\alpha i-\beta l+1}^{\left(\frac{M}{\theta}\right), \tau, m}(t-m \tau)\right.\right.$

$\left.-\nu \lambda_{\beta} k_{n}^{2} G_{2 \alpha,-k-\alpha(i-2)-\beta(1+l)+1}^{\left(\frac{M}{\theta}\right), \tau, m}(t-m \tau)\right]$

$+B^{\prime}{ }_{n}(0)\left[\lambda_{\alpha} G_{2 \alpha,-k-\alpha(i-1)-\beta l+2}^{\left(\frac{M}{\theta}\right), \tau, m}(t-m \tau)+\theta G_{2 \alpha,-k-\alpha i-\beta l+2}^{\left(\frac{M}{\theta}\right), \tau, m}(t-m \tau)\right.$

$\left.+\nu \lambda_{\beta} k_{n}^{2} G_{2 \alpha,-k-\alpha(i-2)-\beta(l+1)+2}^{\left(\frac{M}{\theta}\right), \tau, m}(t-m \tau)\right]$

$-M \int_{0}^{t} g(t-u) H(u-\tau(m+1)) G_{2 \alpha,-k-\alpha(i-2)-\beta l}^{\left(\frac{M}{\theta}\right), \tau, m}(u-\tau(m+1)) d u$

$+\frac{2 c}{k_{n} L} H(t-m \tau)\left[G_{2 \alpha,-k-\alpha(i-2)-\beta l+1}^{\left(\frac{M}{\theta}\right), \tau, m}(t-m \tau)-\lambda_{\alpha} G_{2 \alpha,-k-\alpha(i-1)-\beta l+2}^{\left(\frac{M}{\theta}\right), \tau, m}(t-m \tau)\right.$

$\left.-\theta \Gamma(-\alpha+2) G_{2 \alpha,-k-\alpha i-\beta l+2}^{\left(\frac{M}{\theta}\right), \tau, m}(t-m \tau)\right]$

$+\frac{2 c M}{k_{n} L} H(t-\tau(m+1)) G_{2 \alpha,-k-\alpha(i-2)-\beta l+2}^{\left(\frac{M}{\theta}\right), \tau, m}(t-\tau(m+1))$

$\left.+\frac{2 c}{k_{n} L} \int_{0}^{t} h(t-u) H(u-\tau(m+1)) G_{2 \alpha,-k-\alpha(i-2)-\beta l}^{\left(\frac{M}{\theta}\right), \tau, m}(u-\tau(m+1)) d u\right\}$.

Example 2. We consider the flow of an Oldroyd-B fluid with the initial conditions $\psi_{1}(y)=c, \psi_{2}(y)=0$ and boundary conditions, $\varphi_{1}(t)=c t, \varphi_{2}(t)=0$ where $\mathrm{c}$ is constant. The problem now becomes,

$$
\begin{gathered}
\frac{\partial u(y, t)}{\partial t}+\lambda_{\alpha} D_{t}^{\alpha} u(y, t)=\nu \frac{\partial^{2} u(y, t)}{\partial y^{2}}+\nu \lambda_{\beta} D_{t}^{\beta} \frac{\partial^{2} u(y, t)}{\partial y^{2}}-M u(y, t-\tau) \\
u(y, t)=c, \quad u(0, t)=c t, \quad-\tau \leq t \leq 0, y>0 \\
u_{t}(y, t)=0, \quad u(L, t)=0, \quad 0<\alpha, \beta<\frac{1}{2} .
\end{gathered}
$$

Using the preceding method we obtain,

$$
\begin{aligned}
& B_{n}(t)=\sum_{m=0}^{\infty} \sum_{k, i, j, q \geq 0}^{k+i+j+q=m} \frac{(-1)^{m}}{\left(M \lambda_{\alpha}\right)^{m+1}} \frac{m !\left(-k_{n}{ }^{2} \nu\right)^{i+j} \lambda_{\beta}{ }^{j}}{k ! i ! j !} \\
& \left\{B _ { n } ( 0 ) H ( t - m \tau ) \left[G_{\alpha, \alpha-k-\beta j}^{\left(\frac{M}{\lambda_{\alpha}}\right), \tau, m}(t-m \tau)+\lambda_{\alpha} G_{\alpha,-k-\beta q+1}^{\left(\frac{M}{\lambda_{\alpha}}\right), \tau, m}(t-m \tau)\right.\right. \\
& \left.-\nu \lambda_{\beta} k_{n}{ }^{2} G_{\alpha, \alpha-k-\beta(j+1)+1}^{\left(\frac{M}{\lambda_{\alpha}}\right), \tau, m}(t-m \tau)\right]-M \int_{0}^{t} g(t-u) H(u-\tau(m+1)) G_{\alpha, \alpha-k-\beta j}^{\left(\frac{M}{\lambda_{\alpha}}\right), \tau, m}(u-\tau(m+1)) d u \\
& +\frac{2 c}{k_{n} L} H(t-m \tau)\left[G_{\alpha, \alpha-k-\beta j+1}^{\left(\frac{M}{\lambda_{\alpha}}\right), \tau, m}(t-m \tau)-\lambda_{\alpha} G_{\alpha,-k-\beta j+2}^{\left(\frac{M}{\lambda_{\alpha}}\right), \tau, m}(t-m \tau)\right] \\
& +\frac{2 c M}{k_{n} L} H(t-\tau(m+1)) G_{\alpha, \alpha-k-\beta j+2}^{\left(\frac{M}{\lambda_{\alpha}}\right), \tau, m}(t-\tau(m+1) \\
& \left.+\frac{2 c}{k_{n} L} \int_{0}^{t} h(t-u) H(u-\tau(m+1)) G_{\alpha, \alpha-k-\beta j}^{\left(\frac{M}{\lambda_{\alpha}}\right), \tau, m}(t-m \tau) d u\right\}, \text { after which } W(y, t) \text { and so } u(y, t) \text { may be found. }
\end{aligned}
$$

\section{Conclusion}

In this paper we used a variant of the method of separation of variables to simplify the governing fractionalorder partial differential equations of a generalized viscoelastic Oldroyd-B fluid with constant delay in time to a set of fractional-order ordinary differential equations with homogeneous boundary condition. The Laplace transformation (followed by its inverse) was then employed to obtain the exact solutions of the linear fractional ordinary differential equation. The solutions are given in terms of multivariate Green functions. We found exact solutions for three specific situations illustrated by examples.

\section{References}

1 Podlubny, I. (1998). Fractional differential equations: an introduction to fractional derivatives, fractional differential equations, to methods of their solution and some of their applications. Vol. 198, Elsevier. 
2 Diethelm, K., \& Freed, A.D. (1999). On the solution of nonlinear fractional-order differential equations used in the modeling of viscoplasticity. Springer.

3 Magin, R.L. (2006). Fractional calculus in bioengineering. Begell House Redding.

4 Kilbas, A.A.A., Srivastava, H.M., \& Trujillo, J.J. (2006). Theory and applications of fractional differential equations. Vol. 204, Elsevier Science Limited.

5 Fetecau, C., Fetecau, C., Khan, M., \& Vieru, D. (2008). Decay of a potential vortex in a generalized Oldroyd-B fluid. Applied Mathematics and Computation, 205 (1), 497-506.

6 Khan, M. (2009). The Rayleigh-Stokes problem for an edge in a viscoelastic fluid with a fractional derivative model. Nonlinear Analysis: Real World Applications 10(5), 3190-3195.

7 Nadeem, S. (2007). General periodic flows of fractional Oldroyd-B fluid for an edge. Physics Letters A, $368(3-4), 181-187$.

8 Song, D.Y., \& Jiang, T.Q. (1998). Study on the constitutive equation with fractional derivative for the viscoelastic fluids - Modified Jeffreys model and its application. Rheologica Acta, 37(5), 512-517.

9 Hilfer, R. (2000). Applications of Fractional Calculus in Physics. Edited by HILFER R: Published by World Scientific Publishing Co. Pte. Ltd.

10 Tong, D., Zhang, X., \& Zhang, X. (2009). Unsteady helical flows of a generalized Oldroyd-B fluid. Journal of non-newtonian fluid mechanics, 156(1-2), 75-83.

11 Haitao, Q., \& Mingyu, X. (2009). Some unsteady unidirectional flows of a generalized Oldroyd-B fluid with fractional derivative. Applied Mathematical Modelling, 33(11), 4184-4191.

12 Fetecau, C., Fetecau, C., Kamran, M., \& Vieru, D. (2009). Exact solutions for the flow of a generalized Oldroyd-B fluid induced by a constantly accelerating plate between two side walls perpendicular to the plate. Journal of Non-Newtonian Fluid Mechanics, 156(3), 189-201.

13 Vieru, D., Fetecau, C., \& Fetecau, C. (2008). Flow of a generalized Oldroyd-B fluid due to a constantly accelerating plate. Applied Mathematics and Computation, 201 (1-2), 834-842.

14 Shah, S. H. A. M., Khan, M., \& Qi, H. (2009). Exact solutions for a viscoelastic fluid with the generalized Oldroyd-B model. Nonlinear Analysis: Real World Applications, 10(4), 2590-2599.

15 Qi, H., \& Jin, H. (2009). Unsteady helical flows of a generalized Oldroyd-B fluid with fractional derivative. Nonlinear analysis: real world applications, 10(5), 2700-2708.

16 Zheng, L., Liu, Y., \& Zhang, X. (2011). Exact solutions for MHD flow of generalized Oldroyd-B fluid due to an infinite accelerating plate. Mathematical and Computer Modelling, 54 (1-2), 780-788.

17 Hayat, T., Khan, M., \& Asghar, S. (2007). On the MHD flow of fractional generalized burgers fluid with modified darcy law. Acta Mechanica Sinica, 23(3), 257-261.

18 Heris, M.S., \& Javidi, M. (2017). On fractional backward differential formulas for fractional delay differential equations with periodic and anti-periodic conditions. Applied Numerical Mathematics, 118, $203-220$.

19 Wang, Z., Huang, X., \& Shi, G. (2011). Analysis of nonlinear dynamics and chaos in a fractional order financial system with time delay. Computers and Mathematics with Applications, 62 (3), 1531-1539.

20 Heris, M.S., \& Javidi, M. (2018). On fractional backward differential formulas methods for fractional differential equations with delay. International Journal of Applied and Computational Mathematics, $4(2), 72$.

21 Morgado, M.L., Ford, N.J., \& Lima, P.M. (2013). Analysis and numerical methods for fractional differential equations with delay. Journal of computational and applied mathematics, 252, 159-168.

22 Čermák, J., Horníček, J., \& Kisela, T. (2016). Stability regions for fractional differential systems with a time delay. Communications in Nonlinear Science and Numerical Simulation, 31(1-3), 108-123.

23 Lazarević, M.P., \& Spasić, A.M. (2009). Finite-time stability analysis of fractional order time-delay systems: Gronwall approach. Mathematical and Computer Modelling, 49(3-4), 475-481.

24 Heris, M.S., \& Javidi, M. (2017). On fbdf5 method for delay differential equations of fractional order with periodic and anti-periodic conditions. Mediterranean Journal of Mathematics, $14(3), 134$.

25 Jiang, H., Liu, F., Turner, I., \& Burrage, K. (2012). Analytical solutions for the multi-term time-space caputo-riesz fractional advection-diffusion equations on a finite domain. Journal of Mathematical Analysis and Applications, 389(2), 1117-1127. 


\author{
С. Сагали ${ }^{1}$, Ф.Д. Саи ${ }^{1}$, М. Джавиди ${ }^{2}$, М.Дж. Рад ${ }^{1}$ \\ ${ }^{1}$ Азад Ислам университетінін, Тебриздегі филиаль, Тебриз, Иран; \\ ${ }^{2}$ Тебриз университеті, Тебриз, Иран
}

\title{
Тұтқыр сығынды сұйықтықтар теориясының бөлшек дифференциалдық теңдеуінің аналитикалық шешімі
}

\begin{abstract}
Мақаланың мақсаты - Олдройд-Б сығылмайтын жалпылама сұйықтығын Капуто түріндегі бөлшек туындыларымен кешіктіру арқылы бөлшек дифференциалдық теңдеулердің аналитикалық шешімдерін ұсыну. Айнымалыларды бөлу әдісінің модификациясын қолдана отырып, біртекті емес шекаралық шарттары бар негізгі теңдеу біртекті шекаралық шарттары бар теңдеуге айналады, содан кейін алынған шешімдер Лаплас түрлендірулерінің көмегімен Грин функциялары арқылы көрінеді. Бұл нәтижелер екі жағдайда ұсынылған: бірінші қадамда $0 \leq \alpha, \beta \leq \frac{1}{2}$, ал екінші қадамда $\frac{1}{2} \leq \alpha, \beta \leq 1$, әр қадам үшін 1, 2 Олдройд-Б жалпыланған сұйықтығының стационарлық емес ағымдары үшін, оның ішінде жылжымалы плитасы бар ағын мысалдармен қарастырылды.
\end{abstract}

Kiлm сөздер: Олдройд-Б сұйықтығы, бөлшек ретті жартылай туындылардағы теңдеулер, аналитикалық шешімдер, кешіктірілген дифференциалдық теңдеу, айнымалыларды бөлудің модификацияланған әдісі, Капутоның бөлшек туындылары.

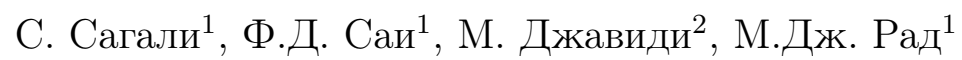 \\ ${ }^{1}$ Филиал Исламского университета Азад в Тебризе, Тебриз, Иран; \\ ${ }^{2}$ Университет Тебриза, Тебриз, Иран
}

\section{Аналитическое решение дробного дифференциального уравнения теории вязкоупругих жидкостей}

Цель данной статьи - представить аналитические решения дробных дифференциальных уравнений с запаздыванием несжимаемой обобщенной жидкости Олдройда-Б с дробными производными типа Капуто. Используя модификацию метода разделения переменных, основное уравнение с неоднородными граничными условиями преобразуется в уравнение с однородными граничными условиями, а полученные решения затем выражаются через функции Грина с помощью преобразований Лапласа. Эти результаты представлены в двух условиях: на первом шаге, когда $0 \leq \alpha, \beta \leq \frac{1}{2}$, а на втором - при $\frac{1}{2} \leq \alpha, \beta \leq 1$. Для каждого шага 1,2 для нестационарных течений обобщенной жидкости Олдройда-Б, включая поток с движущейся пластиной, приведены примеры.

Ключевые слова: жидкость Олдройда-Б, уравнения в частных производных дробного порядка, аналитические решения, дифференциальное уравнение с запаздыванием, модифицированный метод разделения переменных, дробные производные Капуто. 